\title{
Note on anomalous currents for a free theory
}

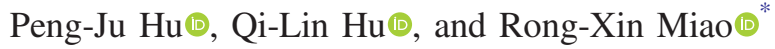 \\ School of Physics and Astronomy, Sun Yat-Sen University, 2 Daxue Road, Zhuhai 519082, China
}

(Received 24 April 2020; accepted 27 May 2020; published 11 June 2020)

\begin{abstract}
Recently it was found that, due to Weyl anomaly, an external magnetic field can induce anomalous currents near a boundary. In this paper, we study anomalous currents for complex scalars and Dirac fields in general dimensions. We develop a perturbation method to calculate Green's function in the spacetime with boundaries. By applying this method, we obtain anomalous currents up to the linear order of magnetic fields in a half space and in a strip. To the best of our knowledge, the results for Dirac fermions and for strips are new. It is remarkable that, unlike the scalars and holographic boundary conformal field theory, the anomalous currents of Dirac fields are independent of boundary conditions in general dimensions. Besides, the currents of Dirac fields are always larger than those of complex scalars. Finally, we find an exact formal expression of the anomalous current in a half space. The result is expressed in momentum integrals, which can be evaluated numerically. We find that the mass suppresses the anomalous currents as expected.
\end{abstract}

DOI: 10.1103/PhysRevD.101.125010

\section{INTRODUCTION}

The anomaly-induced transport is an important phenomenon, which has a wide range of applications [1]. The well-known examples include chiral magnetic effect (CME) [2-6] and chiral vortical effect (CVE) [7-13], which refer to the generation of currents due to an external magnetic field and the rotational motion in the charged fluid, respectively. It is interesting that the CME current is topologically protected and hence nondissipative [1]. Similar to chiral anomaly, Weyl anomaly [14] can also induce anomalous currents in an external electromagnetic field [15-18]. See [19-26] for related works. It is remarkable that a similar mechanism leads to novel Fermi condensations when a background scalar field is turned on [27]. The scalar field can either be the Higgs field in a fundamental theory or the phonon in a condensed matter system.

On the other hand, the boundary effect of quantum field theory is another interesting phenomenon. Famous examples include the Casimir effect [28-30] and topological insulator [31]. Recently, much attention has been drawn on boundary conformal field theory (BCFT) $[32,33]$ and its holographic dual (AdS/BCFT) [34]. Please see [19-22, 35-57] for some recent developments. It is interesting that

\footnotetext{
*miaorx@mail.sysu.edu.cn
}

Published by the American Physical Society under the terms of the Creative Commons Attribution 4.0 International license. Further distribution of this work must maintain attribution to the author(s) and the published article's title, journal citation, and DOI. Funded by SCOAP. a-type anomalies of BCFT/ dCFT can depend on marginal couplings [57].

Weyl anomaly measures the breaking of scaling symmetry of CFT/BCFT due to quantum effects [14]. It is closely related to the UV logarithmic divergent term of effective action [17,21]. As a result, one can derive a key relation [17]

$$
(\delta \mathcal{A})_{\partial M}=\left(\int_{M} \sqrt{|g|}\left\langle J^{\mu}\right\rangle \delta A_{\mu}\right)_{\log \epsilon},
$$

between the renormalized current $\left\langle J^{\mu}\right\rangle$ and the boundary part of the variation of the Weyl anomaly $\mathcal{A}$. Here $A_{\mu}$ is the gauge field and $\epsilon$ denotes the cutoff of the theory. By applying (1), [17,18] find that, due to the Weyl anomaly,

$$
\mathcal{A}=\int_{M} \sqrt{|g|} \frac{\beta}{2} F^{\mu \nu} F_{\mu \nu}
$$

an external electromagnetic field can induce an universal current

$$
\left\langle J^{\mu}\right\rangle=\frac{-2 \beta F^{\mu \nu} n_{\nu}}{x}+\cdots, \quad x \sim 0,
$$

near the boundary, where $\beta$ is the beta function, $F^{\mu \nu}$ are the field strength, $x$ is the proper distance to the boundary, $n_{i}$ are the normal vectors and ... denotes higher order terms in $O(x)$. Note that there are boundary contributions to the current, which can cancel the "bulk" divergence of (3) and make finite the total current [17]. It is remarkable that the leading term of the anomalous current (3) is universal in four dimensions. It is independent of the 
boundary conditions (BC), the temperature, and the details of theories. What is more, it applies to not only conformal field theory but also the general quantum field theory [17]. In higher dimensions, the anomalous current is expected to take the following form [17]:

$$
\left\langle J_{d}^{\mu}\right\rangle=\frac{b_{d} F^{\mu \nu} n_{\nu}}{x^{d-3}}+\cdots, \quad x \sim 0,
$$

where $b_{d}$ are central charges of Weyl anomaly and $d$ denote the dimensions. Note that $b_{d}$ depend on boundary conditions in dimensions other than four.

In this paper, we investigate the anomalous current in a spacetime with a boundary. The previous works $[17,18]$ mainly discuss the general characteristics of anomalous currents. In particular, they focus on the region close to the boundary. In this paper, we study more specific examples such as complex scalars and Dirac fermions, and try to explore the anomalous current in the full region of the system. By applying Green's function method, we derive the anomalous currents for free theories up to the linear order of external magnetic field in a half space and in a strip. Our results agree with the work of [58] for complex scalars in a half space. To the best of our knowledge, the anomalous currents for Dirac fermions and for strips are new. Our results are exact in the size of strip, and hence work well in the full region of a strip. We also obtain an exact formal expression of the anomalous current for complex scalars in a half space. The formal formula is expressed in momentum integrals, which can be evaluated numerically. Let us summarize the properties of anomalous currents for free theories below.

(1) Unlike the holographic BCFT [18] and complex scalars, the anomalous currents of Dirac fields are independent of BCs in general dimensions.

(2) The anomalous currents of complex scalars have different signs for Dirichlet boundary condition (DBC) and Neumann boundary condition (NBC) in dimensions higher than four. See Fig. 2 for example.

(3) The anomalous currents of Dirac fields are larger than those of complex scalars in four dimensions. See Fig. 3 for example.

(4) The mass suppresses the anomalous current and the current approaches zero far away from the boundary. See Fig. 5 for example.

The paper is organized as follows. In Sec. II, we develop a perturbation method to calculate Green's function. Our method includes only a bulk integral, which is slightly different from that of $[59,60]$. In Sec. III, by applying the perturbation method, we derive the anomalous currents for a complex scalar and a Dirac field in a half space in general dimensions. In Sec. IV, we study the anomalous current in a strip. In Sec. V, we obtain a formal expression of the anomalous current in a half space. Finally, we conclude with discussions in Sec. VI. We use conventions of [61] and the signature of metric is $(1,-1, \ldots,-1)$.

\section{GREEN'S FUNCTION}

Green's function is a powerful tool to calculate expectation value of stress-energy tensors and currents near a boundary $[59,60]$. Usually, Green's function is expressed as a boundary integral for BCFT $[59,60]$. Here we develop a slightly different approach where Green's function includes only bulk integrals. Our approach has the advantage that the $n$th term of a series of Green's functions is of order $O\left(B^{n}\right)$

$$
G=\sum_{n=0}^{\infty} G_{n}, \quad G_{n} \sim O\left(B^{n}\right)
$$

where $B$ denotes the magnetic field or other perturbation parameters. As a result, to derive the leading term of anomalous currents of $O(B)$, we only need to calculate one term $G_{1}$ in Green's function. To illustrate our approach, let us take complex scalars and Dirac fields as examples below.

\section{A. Complex scalar}

Let us start with the action of free complex scalars in a curved spacetime

$$
I=\int_{M} d x^{d} \sqrt{|g|} D_{\nu} \phi\left(D^{\nu} \phi\right)^{*},
$$

where $d$ is the dimension of spacetime and $D_{\mu}=$ $\nabla_{\mu}-i e A_{\mu}$ is the covariant derivative. For simplicity, we set $e=1$ in this paper. Green's function satisfies equation of motion $(\mathrm{EOM})$

$$
D^{\mu} D_{\mu} G\left(x, x^{\prime}\right)=\left(\nabla^{\mu} \nabla_{\mu}+E\right) G\left(x, x^{\prime}\right)=\delta\left(x, x^{\prime}\right),
$$

where we take $E=\left(-2 i A^{\mu} \nabla_{\mu}-i \nabla^{\mu} A_{\mu}-A^{\mu} A_{\mu}\right)$ as perturbations. One can impose either DBC

$$
\left.G\left(x, x^{\prime}\right)\right|_{\partial M}=0,
$$

or NBC

$$
\left.D_{n} G\left(x, x^{\prime}\right)\right|_{\partial M}=0,
$$

on the boundary $\partial M$. Here $n$ denotes the normal direction.

We split Green's function into the background $G_{0}$ and a correction $G_{c}$

$$
G=G_{0}+G_{c},
$$

where $G_{0}$ obeys EOM

$$
\nabla^{\mu} \nabla_{\mu} G_{0}\left(x, x^{\prime}\right)=\delta\left(x, x^{\prime}\right)
$$


together with either DBC

$$
\left.G_{0}\right|_{\partial M}=0
$$

or NBC

$$
\left.\nabla_{n} G_{0}\right|_{\partial M}=0 .
$$

Applying Green's formula, we have

$$
\begin{array}{rl}
\int_{M} & d x^{d} \sqrt{|g|}\left[G_{c}\left(x^{\prime}, x\right) D^{\mu} D_{\mu} G\left(x, x^{\prime \prime}\right)\right. \\
& \left.-G_{c}\left(x^{\prime}, x\right) \overleftarrow{D}^{* \mu} \overleftarrow{D}_{\mu}^{*} G\left(x, x^{\prime \prime}\right)\right] \\
= & \int_{\partial M} d y^{d-1} \sqrt{|h|}\left[G_{c}\left(x^{\prime}, x\right) D_{n} G\left(x, x^{\prime \prime}\right)\right. \\
& \left.-G_{c}\left(x^{\prime}, x\right) \overleftarrow{D}_{n}^{*} G\left(x, x^{\prime \prime}\right)\right]
\end{array}
$$

where $\overleftarrow{D}_{\mu}^{*}=\overleftarrow{\nabla}_{\mu}+i A_{\mu}$ means acting on the left. We choose the gauge $A_{n}=0$ so that $D_{n}=D_{n}^{*}=\nabla_{n}$ on the boundary. Imposing either DBC (8), (12) or NBC (9), (13), we find that the boundary terms of (14) vanish. From EOM (7), (11), we derive

$$
G_{c}\left(x^{\prime}, x\right) \overleftarrow{D}^{* \mu} \overleftarrow{D}_{\mu}^{*}=-G_{0}\left(x^{\prime}, x\right) \overleftarrow{E}^{*}
$$

where

$$
\overleftarrow{E}^{*}=\left(2 i \overleftarrow{\nabla}_{\mu} A^{\mu}+i \nabla^{\mu} A_{\mu}-A^{\mu} A_{\mu}\right)
$$

Substituting (7) and (15) into (14) and noting that the boundary terms vanishing due to BCs, we obtain a key formula

$$
G_{c}\left(x^{\prime}, x^{\prime \prime}\right)=-\int_{M} d x^{d} \sqrt{|g|}\left[G_{0}\left(x^{\prime}, x\right) \overleftarrow{E}^{*}(x) G\left(x, x^{\prime \prime}\right)\right] .
$$

Unlike [59,60], there are only bulk integrals in $G_{c}$. From (17), we can calculate $G_{c}$ perturbatively

$$
\begin{aligned}
G_{c}\left(x^{\prime}, x^{\prime \prime}\right)= & -\int_{M} d x^{d} \sqrt{|g|} G_{0}\left(x^{\prime}, x\right) \overleftarrow{E}^{*}(x) G_{0}\left(x, x^{\prime \prime}\right) \\
& +\int_{M} d x^{d} \sqrt{|g|} \int_{M_{1}} d x_{1}^{d} \sqrt{\left|g_{1}\right|} G_{0}\left(x^{\prime}, x\right) \overleftarrow{E}^{*}(x) \\
& \times G_{0}\left(x, x_{1}\right) \overleftarrow{E}^{*}\left(x_{1}\right) G_{0}\left(x_{1}, x^{\prime \prime}\right)+\cdots
\end{aligned}
$$

where the $n$th line of (18) is of order $O\left(E^{n}\right)$.

\section{B. Dirac field}

Now let us turn to study Green's function of Dirac fields. The action of free Dirac fields in a curved spacetime is given by

$$
I=\int_{M} d x^{d} \sqrt{|g|} \bar{\Psi} i \gamma^{\mu} D_{\mu} \Psi
$$

where $\bar{\Psi}=\Psi^{+} \gamma^{0}$ and $D_{\mu}=\nabla_{\mu}-i A_{\mu}$. Green's function obeys EOM

$$
i \gamma^{\mu} D_{\mu} S\left(x, x^{\prime}\right)=\delta\left(x, x^{\prime}\right)
$$

where $\delta\left(x, x^{\prime}\right)=\delta^{d}\left(x-x^{\prime}\right) / \sqrt{|g|}$. We impose bag boundary condition (BBC)

$$
\left.\Pi_{-} S\left(x, x^{\prime}\right)\right|_{\partial M}=0,
$$

where $\Pi_{ \pm}=(1 \pm \chi) / 2$ are projection operators and $\chi$ satisfy [33]

$$
\chi \gamma_{n}=-\gamma_{n} \bar{\chi}, \quad \chi \gamma_{a}=\gamma_{a} \bar{\chi}, \quad \chi^{2}=\bar{\chi}^{2}=1,
$$

where $\bar{\chi}=\gamma^{0} \chi^{+} \gamma^{0}$ and $n(a)$ denote the normal (tangent) directions. From BBC (21), (22), we can derive

$$
\left.S\left(x^{\prime \prime}, x\right) \gamma_{n} S\left(x, x^{\prime}\right)\right|_{\partial M}=0 .
$$

We split Green's function into a background $S_{0}$ and a correction $S_{c}$

$$
S=S_{0}+S_{c}
$$

where $S_{0}$ obeys EOM

$$
i \gamma^{\mu} \nabla_{\mu} S_{0}\left(x, x^{\prime}\right)=\delta\left(x, x^{\prime}\right)
$$

together with $\mathrm{BBC}$

$$
\left.\Pi_{-} S_{0}\left(x, x^{\prime}\right)\right|_{\partial M}=0 .
$$

From (21), (22), (26), we have

$$
\left.S_{A}\left(x^{\prime \prime}, x\right) \gamma_{n} S_{B}\left(x, x^{\prime}\right)\right|_{\partial M}=0,
$$

where $S_{A, B}$ denote $S, S_{0}, S_{c}$.

Applying Green's formula for Dirac fields, we have

$$
\begin{aligned}
\int_{M} d x^{d} \sqrt{|g|}\left[S_{c}\left(x^{\prime}, x\right) i \gamma^{\mu}\left(\vec{\nabla}_{\mu}-i A_{\mu}\right) S\left(x, x^{\prime \prime}\right)\right. \\
\left.\quad+S_{c}\left(x^{\prime}, x\right) i \gamma^{\mu}\left(\overleftarrow{\nabla}_{\mu}+i A_{\mu}\right) S\left(x, x^{\prime \prime}\right)\right] \\
=\int_{\partial M} d x^{d-1} \sqrt{|h|}\left[S_{c}\left(x^{\prime}, x\right) i \gamma_{n} S\left(x, x^{\prime \prime}\right)\right]
\end{aligned}
$$

Note that the boundary term of (28) vanishes due to (27). Applying EOM (20), (25), we derive

$$
S_{c}\left(x^{\prime}, x\right) i \gamma^{\mu}\left(\overleftarrow{\nabla}_{\mu}+i A_{\mu}\right)=S_{0}\left(x^{\prime}, x\right) \gamma^{\mu} A_{\mu}
$$


Now (28) can be simplified as

$$
S_{c}\left(x^{\prime}, x^{\prime \prime}\right)=-\int_{M} d x^{d} \sqrt{|g|}\left[S_{0}\left(x^{\prime}, x\right) \gamma^{\mu}(x) A_{\mu}(x) S\left(x, x^{\prime \prime}\right)\right] .
$$

From (30), we can calculate $S_{c}$ perturbatively

$$
\begin{aligned}
S_{c}\left(x^{\prime}, x^{\prime \prime}\right)= & -\int_{M} d x^{d} \sqrt{|g|} S_{0}\left(x^{\prime}, x\right) \gamma^{\mu} A_{\mu}(x) S_{0}\left(x, x^{\prime \prime}\right) \\
& +\int_{M} d x^{d} \sqrt{|g|} \int_{M_{1}} d x_{1}^{d} \sqrt{\left|g_{1}\right|} S_{0}\left(x^{\prime}, x\right) \gamma^{\mu}(x) \\
& \times A_{\mu}(x) S_{0}\left(x, x_{1}\right) \gamma^{\mu}\left(x_{1}\right) A_{\mu}\left(x_{1}\right) S_{0}\left(x_{1}, x^{\prime \prime}\right)+\cdots
\end{aligned}
$$

where the $n$th line of (31) is of order $O\left(A^{n}\right)$.

Now we finish the perturbative derivations of Green's function for complex scalars (18) and Dirac fields (31).

\section{CURRENT IN A HALF SPACE}

In this section we calculate the anomalous current for complex scalars and Dirac fields in a half space. For simplicity, we focus on the half space $x \geq 0$ with a constant magnetic field parallel to the boundary. We have $x^{\mu}=\left(t, x, y_{a}\right)=\left(t, x, y_{1}, \ldots, y_{d-2}\right), A_{\mu}=(0,0$, $B x, 0, \ldots, 0)$ and $g_{\mu \nu}=\eta_{\mu \nu}=\operatorname{diag}(1,-1, \ldots,-1)$.

\section{A. Complex scalar}

Green's function of the complex scalar is given by [59]

$$
G\left(x, x^{\prime}\right)=i\left\langle T \phi(x) \phi^{*}\left(x^{\prime}\right)\right\rangle
$$

where $T$ is the time-ordering symbol. The nonrenormalized current is defined by

$$
\hat{J}_{\mu}(x)=\frac{\delta I_{\mathrm{eff}}}{\sqrt{|g|} \delta A^{\mu}}=\lim _{x^{\prime} \rightarrow x}\left(D_{\mu}-D_{\mu^{\prime}}^{*}\right) G\left(x, x^{\prime}\right)
$$

which is divergent generally. Here $I_{\text {eff }}$ denotes the effective action. To get the renormalized current $J_{\mu}$, one should subtract the reference current without boundaries

$$
J_{\mu}(x)=\lim _{x^{\prime} \rightarrow x}\left(D_{\mu}-D_{\mu^{\prime}}^{*}\right)\left(G\left(x, x^{\prime}\right)-\bar{G}\left(x, x^{\prime}\right)\right),
$$

where $\bar{G}$ is a reference Green's function in the spacetime without boundaries. By using (18), we can obtain $G$ and $\bar{G}$ as

$$
\begin{aligned}
& G\left(x^{\prime}, x^{\prime \prime}\right)=G_{0}\left(x^{\prime}, x^{\prime \prime}\right)+2 i B \int_{0}^{\infty} d x \int_{-\infty}^{\infty} d t d y^{d-2} G_{0}\left(x^{\prime}, x\right) x \overleftarrow{\partial}_{y_{1}} G_{0}\left(x, x^{\prime \prime}\right)+O\left(B^{2}\right), \\
& \bar{G}\left(x^{\prime}, x^{\prime \prime}\right)=\bar{G}_{0}\left(x^{\prime}, x^{\prime \prime}\right)+2 i B \int_{-\infty}^{\infty} d x \int_{-\infty}^{\infty} d t d y^{d-2} \bar{G}_{0}\left(x^{\prime}, x\right) x \overleftarrow{\partial}_{y_{1}} \bar{G}_{0}\left(x, x^{\prime \prime}\right)+O\left(B^{2}\right) .
\end{aligned}
$$

Note that the integral regions of $x$ are different for $G$ and $\bar{G}$. Here $G_{0}$ and $\bar{G}_{0}$ are Green's functions without external magnetic fields

$$
\begin{gathered}
G_{0}\left(x^{\prime}, x^{\prime \prime}\right)=\frac{\Gamma\left(\frac{d}{2}-1\right)}{4 \pi^{d / 2}}\left(\frac{i}{\left[\left(x^{\prime}-x^{\prime \prime}\right)^{2}+\left(y_{a}^{\prime}-y_{a}^{\prime \prime}\right)^{2}-\left(t^{\prime}-t^{\prime \prime}\right)^{2}\right]^{\frac{d-2}{2}}}+\chi \frac{i}{\left[\left(x^{\prime}+x^{\prime \prime}\right)^{2}+\left(y_{a}^{\prime}-y_{a}^{\prime \prime}\right)^{2}-\left(t^{\prime}-t^{\prime \prime}\right)^{2}\right]^{\frac{d-2}{2}}}\right), \\
\bar{G}_{0}\left(x^{\prime}, x^{\prime \prime}\right)=\frac{\Gamma\left(\frac{d}{2}-1\right)}{4 \pi^{d / 2}} \frac{i}{\left[\left(x^{\prime}-x^{\prime \prime}\right)^{2}+\left(y_{a}^{\prime}-y_{a}^{\prime \prime}\right)^{2}-\left(t^{\prime}-t^{\prime \prime}\right)^{2}\right]^{\frac{d-2}{2}}},
\end{gathered}
$$

where $\chi=-1$ for DBC and $\chi=1$ for NBC. One can check that $G_{0}$ (37) satisfy EOM (11) and BCs (12), (13).

To do the integral along $t$, it is more convenient to perform the Wick rotation $t=-i t_{E}$. Substituting (35)-(38) into (34) and performing the Wick rotation, we get

$$
J_{y_{1}}=\chi^{1-d} \pi^{-\frac{d}{2}} \Gamma\left(\frac{d}{2}-1\right) B x^{3-d}+B \int_{0}^{\infty} d x^{\prime} \int_{0}^{\infty} d r\left(f_{1}+\chi f_{2}\right)+O\left(B^{2}\right),
$$

where

$$
f_{1}=\frac{(2-d) r^{d-2} \Gamma\left(\frac{d}{2}-1\right)^{2} x^{\prime}\left((d-3) r^{2}+(d-1)\left(x+x^{\prime}\right)^{2}\right)}{4 \pi^{\frac{1}{2}(d+1)} \Gamma\left(\frac{d+1}{2}\right)\left(r^{2}+\left(x^{\prime}+x\right)^{2}\right)^{d}}
$$




$$
\begin{aligned}
f_{2}= & \frac{x^{\prime}(2-d) \pi^{-\frac{d}{2}-\frac{1}{2}} r^{d-2} \Gamma\left(\frac{d}{2}-1\right)^{2}}{4 \Gamma\left(\frac{d+1}{2}\right)\left(r^{2}+\left(x-x^{\prime}\right)^{2}\right)^{\frac{d}{2}+1}\left(r^{2}+\left(x^{\prime}+x\right)^{2}\right)^{\frac{d}{2}+1}}\left[(d-3) r^{6}+(3 d-7) r^{4}\left(x^{2}+x^{\prime 2}\right)\right. \\
& \left.+r^{2}\left((3 d-5) x^{4}+(3 d-5) x^{\prime 4}+2(1-3 d) x^{2} x^{\prime 2}\right)+(d-1)\left(x^{2}-x^{\prime 2}\right)^{2}\left(x^{2}+x^{\prime 2}\right)\right]
\end{aligned}
$$

Here $r^{2}=y_{a}^{2}+t_{E}^{2}, \chi^{2}=1$ and we have performed angle integrals above. After the radial integration, we get

$$
\int_{0}^{\infty} d r\left(f_{1}+\chi f_{2}\right)= \begin{cases}\frac{2^{2-d} \pi^{-\frac{d}{2}} \Gamma\left(\frac{d}{2}\right) x^{\prime}\left(\left(x^{\prime}\right)^{d-1}+\chi\left(x^{\prime}+x\right)^{d-1}\right)}{(1-d)\left(x^{\prime}\right)^{d-1}\left(x^{\prime}+x\right)^{d-1}}, & x^{\prime}>x \\ \frac{2^{2-d} \pi^{-\frac{d}{2}} \Gamma\left(\frac{d}{2}\right) x^{\prime}\left(x^{d-1}+\chi\left(x^{\prime}+x\right)^{d-1}\right)}{(1-d) x^{d-1}\left(x^{\prime}+x\right)^{d-1}}, & x^{\prime}<x .\end{cases}
$$

Note that the integrals are different for $x^{\prime}>x$ and $x^{\prime}<x$. Substituting (42) into (39) and integrating along $x^{\prime}$, we finally obtain the anomalous current

$$
J_{y_{1}}=\frac{\pi^{-\frac{d}{2}} \Gamma\left(\frac{d}{2}-1\right)}{2^{d}(d-3)(1-d)}(2-(d-4)(d-1) \chi) \frac{B}{x^{d-3}}+O\left(B^{2}\right)
$$

which agree with the results of [58] derived by the heat-kernel method. Recall that $\chi=1(\chi=-1)$ for NBC (DBC). The other components of currents vanish. Some comments are in order. First, the anomalous current depends on BCs and has different sign for different BCs in dimensions other than four. Second, it is remarkable that the current is independent of BC in four dimensions

$$
J_{4 d}^{\mu}=\delta_{y_{1}}^{\mu} \frac{B}{24 \pi^{2} x}+O\left(B^{2}\right),
$$

which agrees with the result (3) $[17,18]$. Note that $J^{y_{1}}=-J_{y_{1}}$ in our conventions. Third, in the above calculations, we have assumed $d>3$. After the analytical extension, the result (43) works well for DBC in two and three dimensions. However, this is not the case for NBC. In dimensions lower than four, we have

$$
\begin{gathered}
J_{2 d}^{\mu}=\delta_{y_{1}}^{\mu} \begin{cases}\frac{B x}{2 \pi}+O\left(B^{2}\right), & \mathrm{DBC}, \\
\frac{2 B x}{\pi(2-d)}+\frac{B x\left(4 \log (x)+2 \gamma-1+\log \left(16 \pi^{2}\right)\right)}{2 \pi}+O\left(d-2, B^{2}\right), & \mathrm{NBC},\end{cases} \\
J_{3 d}^{\mu}=\delta_{y_{1}}^{\mu} \begin{cases}\frac{B}{16 \pi}+O\left(B^{2}\right), & \mathrm{DBC}, \\
\frac{B}{4 \pi(d-3)}-\frac{B\left(4 \log (x)+3+\log \left(16 \pi^{2}\right)-2 \psi^{(0)}\left(\frac{1}{2}\right)\right)}{16 \pi}+O\left(d-3, B^{2}\right), & \mathrm{NBC},\end{cases}
\end{gathered}
$$

where $\gamma$ is the Euler gamma function and $\psi$ is the polygamma function. One may regularize the currents for NBC by

$$
\begin{aligned}
& J_{2 d}^{\mu}=\lim _{\epsilon \rightarrow 0} \frac{J^{\mu}(d=2+\epsilon)+J^{\mu}(d=2-\epsilon)}{2}, \\
& J_{3 d}^{\mu}=\lim _{\epsilon \rightarrow 0} \frac{J^{\mu}(d=3+\epsilon)+J^{\mu}(d=3-\epsilon)}{2} .
\end{aligned}
$$

In this way, the divergences of (45), (46) cancel and we get finite currents for NBC in two and three dimensions. We leave a careful discussion of the lower-dimensional currents to future work.

\section{B. Dirac field}

Let us go on to discuss the anomalous current for the Dirac field. Similarly, we have $A_{\mu}=(0,0, B x, 0, \ldots, 0)$. Without loss of generality, we choose the chiral bag boundary condition [62]

$$
\left.\Pi_{-} \Psi\right|_{\partial M}=\left.\frac{1+i e^{i \theta \gamma_{5}} \gamma^{n}}{2} \Psi\right|_{\partial M}=0
$$

where $\theta$ is a constant and $n$ denotes the normal direction, i.e., $\gamma^{n}=\gamma^{1}$. Equivalently, we have chosen

$$
\chi=-i e^{i \theta \gamma_{5}} \gamma^{n}
$$


One can check that it satisfies the conditions (22). Note that (49) reduces to the usual bag boundary condition when $\theta=0, \pi$. Note also that since $\gamma_{5}$ is not well defined in odd dimensions, we set $\theta=0, \pi$ so that the boundary condition (49) becomes $\left.\left(1 \pm i \gamma^{n}\right) \Psi\right|_{\partial M}=0$ in odd dimensions.

The Feynman Green's function of the Dirac field is given by [61]

$$
S\left(x, x^{\prime}\right)=-i\left\langle T \Psi(x) \bar{\Psi}\left(x^{\prime}\right)\right\rangle
$$

from which one can derive the current

$$
J^{\mu}=-i \lim _{x^{\prime} \rightarrow x} \operatorname{Tr}\left[\gamma^{\mu}\left(S\left(x, x^{\prime}\right)-\bar{S}\left(x, x^{\prime}\right)\right)\right]
$$

where we have subtracted the reference Green's function $\bar{S}$ without boundaries. From the key formula (31), we get

$$
\begin{aligned}
& S\left(x^{\prime}, x^{\prime \prime}\right)=S_{0}\left(x^{\prime}, x^{\prime \prime}\right)-B \int_{0}^{\infty} d x \int_{-\infty}^{\infty} d t d y^{d-2} S_{0}\left(x^{\prime}, x\right) \gamma^{2} x S_{0}\left(x, x^{\prime \prime}\right)+O\left(B^{2}\right), \\
& \bar{S}\left(x^{\prime}, x^{\prime \prime}\right)=\bar{S}_{0}\left(x^{\prime}, x^{\prime \prime}\right)-B \int_{-\infty}^{\infty} d x \int_{-\infty}^{\infty} d t d y^{d-2} \bar{S}_{0}\left(x^{\prime}, x\right) \gamma^{2} x \bar{S}_{0}\left(x, x^{\prime \prime}\right)+O\left(B^{2}\right),
\end{aligned}
$$

where $\gamma^{2}=\gamma^{y_{1}}$ and

$$
\begin{gathered}
S_{0}\left(x^{\prime}, x^{\prime \prime}\right)=\frac{(d-2) \Gamma\left(\frac{d-2}{2}\right)}{4 \pi^{\frac{d}{2}}}\left(\frac{\gamma^{0}\left(t^{\prime}-t^{\prime \prime}\right)-\gamma^{1}\left(x^{\prime}-x^{\prime \prime}\right)-\gamma^{a}\left(y_{a}^{\prime}-y_{a}^{\prime \prime}\right)}{\left[\left(x^{\prime}-x^{\prime \prime}\right)^{2}+\left(y_{a}^{\prime}-y_{a}^{\prime \prime}\right)^{2}-\left(t^{\prime}-t^{\prime \prime}\right)^{2}\right]^{\frac{d}{2}}}+\chi \cdot \frac{\gamma^{0}\left(t^{\prime}-t^{\prime \prime}\right)-\gamma^{1}\left(-x^{\prime}-x^{\prime \prime}\right)-\gamma^{a}\left(y_{a}^{\prime}-y_{a}^{\prime \prime}\right)}{\left[\left(x^{\prime}+x^{\prime \prime}\right)^{2}+\left(y_{a}^{\prime}-y_{a}^{\prime \prime}\right)^{2}-\left(t^{\prime}-t^{\prime \prime}\right)^{2}\right]^{\frac{d}{2}}}\right), \\
\bar{S}_{0}\left(x^{\prime}, x^{\prime \prime}\right)=\frac{(d-2) \Gamma\left(\frac{d-2}{2}\right)}{4 \pi^{\frac{d}{2}}} \frac{\gamma^{0}\left(t^{\prime}-t^{\prime \prime}\right)-\gamma^{1}\left(x^{\prime}-x^{\prime \prime}\right)-\gamma^{a}\left(y_{a}^{\prime}-y_{a}^{\prime \prime}\right)}{\left[\left(x^{\prime}-x^{\prime \prime}\right)^{2}+\left(y_{a}^{\prime}-y_{a}^{\prime \prime}\right)^{2}-\left(t^{\prime}-t^{\prime \prime}\right)^{2}\right]^{\frac{d}{2}}} .
\end{gathered}
$$

Substituting (53)-(56) into (52) and performing the Wick rotation, we derive

$$
J^{y_{1}}=B \int_{0}^{\infty} d x^{\prime} \int_{0}^{\infty} d r \frac{r^{d-2} \Gamma\left(\frac{d}{2}\right)^{2} x^{\prime} 2^{\left[\frac{d}{2}\right]-1}\left((d-3) r^{2}+(d-1)\left(x^{\prime}+x\right)^{2}\right)}{\pi^{\frac{d+1}{2}} \Gamma\left(\frac{d+1}{2}\right)\left(r^{2}+\left(x^{\prime}+x\right)^{2}\right)^{d}}+O\left(B^{2}\right),
$$

where [] denotes the integer part. After the integrals along $x^{\prime}$ and $r$, we obtain the anomalous current in a half space for Dirac fields

$$
J^{\mu}=\delta_{y_{1}}^{\mu} \frac{\pi^{-\frac{d}{2}} \Gamma\left(\frac{d}{2}\right) 2^{\left[\frac{d}{2}\right]-d+1}}{(d-3)(d-1)} \frac{B}{x^{d-3}}+O\left(B^{2}\right) .
$$

To the best of our knowledge, this result is new. It is remarkable that the leading term of current (58) is independent of choices of BCs (49), that different chiral angles $\theta$ yield the same current. This is quite different from the currents of complex scalars (43) and holographic BCFT [18] which depend on BCs. To end this section, let us list the currents for Dirac fermions (58) in four dimensions

$$
J_{4 d}^{\mu}=\delta_{y_{1}}^{\mu} \frac{B}{6 \pi^{2} x}+O\left(B^{2}\right),
$$

in two dimensions

$$
J_{2 d}^{\mu}=-\delta_{y_{1}}^{\mu} \frac{B x}{\pi}+O\left(B^{2}\right)
$$

and in three dimensions

$$
\begin{aligned}
J_{3 d}^{\mu}= & \delta_{y_{1}}^{\mu}\left(\frac{B}{8 \pi(d-3)}-\frac{B\left(2 \log (x)+1+\log (2 \pi)-\psi^{(0)}\left(\frac{3}{2}\right)\right)}{16 \pi}\right) \\
& +O\left((d-3)^{1}, B^{2}\right) .
\end{aligned}
$$

It is interesting that (58) works well in two dimensions. Note that since there is no magnetic field in two dimensions, $B$ should be understood as the electric field and $J_{2 d}^{y_{1}}$ should be understood as charge density in (60). Similar to the case of scalar, the formula (58) does not work well in three dimensions. One can regularize the $3 \mathrm{~d}$ current in the same way as (48). We leave a careful study of the $3 \mathrm{~d}$ current to future work.

\section{CURRENT IN A STRIP}

We have investigated the anomalous current in a half space $0 \leq x$. Now let us go on to study the anomalous current in a strip $0 \leq x \leq L$. For simplicity, we mainly focus on four dimensions in this section. 


\section{A. Complex scalar}

Let us first discuss the complex scalar. The approach is similar to that of Sec. III A. Applying (18), we get Green's function

$$
G\left(x^{\prime}, x^{\prime \prime}\right)=G_{0}\left(x^{\prime}, x^{\prime \prime}\right)+2 i B \int_{0}^{L} d x \int_{-\infty}^{\infty} d t d y^{d-2} G_{0}\left(x^{\prime}, x\right) x \overleftarrow{\partial}_{y_{1}} G_{0}\left(x, x^{\prime \prime}\right)+O\left(B^{2}\right)
$$

with the background one

$$
\begin{aligned}
G_{0}\left(x^{\prime}, x^{\prime \prime}\right)= & \frac{\Gamma\left(\frac{d}{2}-1\right)}{4 \pi^{d / 2}} \sum_{m=-\infty}^{\infty}\left(\frac{i}{\left[\left(x^{\prime}-x^{\prime \prime}+2 L m\right)^{2}+\left(y_{a}^{\prime}-y_{a}^{\prime \prime}\right)^{2}-\left(t^{\prime}-t^{\prime \prime}\right)^{2}\right]^{\frac{d-2}{2}}}\right. \\
& \left.+\chi \frac{i}{\left[\left(x^{\prime}+x^{\prime \prime}+2 L m\right)^{2}+\left(y_{a}^{\prime}-y_{a}^{\prime \prime}\right)^{2}-\left(t^{\prime}-t^{\prime \prime}\right)^{2}\right]^{\frac{d-2}{2}}}\right)
\end{aligned}
$$

which can be derived by the mirror method. Note that there are infinite images for two parallel mirrors, and each image corresponds to one $m$ of (63). The reference Green's functions are still given by (36), (38). To simplify the deduction, we rewrite the reference Green's function (36) as

$$
\bar{G}\left(x^{\prime}, x^{\prime \prime}\right)=\bar{G}_{0}\left(x^{\prime}, x^{\prime \prime}\right)+O\left(B^{2}\right)+2 i B \sum_{m=-\infty}^{\infty} \int_{0}^{L} d x \int_{-\infty}^{\infty} d t d y^{d-2} \bar{G}_{0}\left(x^{\prime}, x+m L\right)(x+m L) \overleftarrow{\partial}_{y_{1}} \bar{G}_{0}\left(x+m L, x^{\prime \prime}\right)
$$

where $\bar{G}_{0}$ is given by (38). Now let us focus on four dimensions $d=4$. Substituting (62), (63), (64) into (34) and performing the Wick rotation, we get

$$
J_{y_{1}}=\sum_{\bar{m} \neq 0} \frac{B x}{8 \pi^{2} \bar{m}^{2} L^{2}}+\sum_{m=-\infty}^{\infty} \frac{\chi B x}{8 \pi^{2}(L m+x)^{2}}+B \int_{0}^{L} d x^{\prime} \int_{0}^{\infty} d r\left(g_{1}+g_{2}\right)+O\left(B^{2}\right),
$$

where

$$
\begin{gathered}
g_{1}=\sum_{m=-\infty}^{\infty} \frac{r^{2}\left(L m+x^{\prime}\right)\left(3\left(L m+x^{\prime}-x\right)^{2}+r^{2}\right)}{3 \pi^{3}\left(\left(L m+x^{\prime}-x\right)^{2}+r^{2}\right)^{4}} \\
g_{2}=\frac{r^{2} x^{\prime}}{3 \pi^{3}} \sum_{m_{1}, m_{2}=-\infty}^{\infty}\left[-2 r^{2}\left(\frac{\chi}{\left(A_{1}^{2}+r^{2}\right)^{2}}+\frac{1}{\left(A_{2}^{2}+r^{2}\right)^{2}}\right)\left(\frac{\chi}{\left(B_{1}^{2}+r^{2}\right)^{2}}+\frac{1}{\left(B_{2}^{2}+r^{2}\right)^{2}}\right)\right. \\
\left.+\left(4 r^{2}\left(\frac{\chi}{\left(A_{1}^{2}+r^{2}\right)^{3}}+\frac{1}{\left(A_{2}^{2}+r^{2}\right)^{3}}\right)-\frac{3 \chi}{\left(A_{1}^{2}+r^{2}\right)^{2}}-\frac{3}{\left(A_{2}^{2}+r^{2}\right)^{2}}\right)\left(\frac{\chi}{B_{1}^{2}+r^{2}}+\frac{1}{B_{2}^{2}+r^{2}}\right)\right]
\end{gathered}
$$

with $A_{1}=2 L m_{1}+x+x^{\prime}, A_{2}=2 L m_{1}+x-x^{\prime}, B_{1}=2 L m_{2}+x+x^{\prime}$, and $B_{2}=2 L m_{2}-x+x^{\prime}$.

The first two terms of (65) are due to the leading term of Green's function $\left(G_{0}-\bar{G}_{0}\right)$. After the sum, they yield

$$
j_{1}=\frac{B x\left(3 \chi \csc ^{2}\left(\frac{\pi x}{L}\right)+1\right)}{24 L^{2}} .
$$

Let us go on to consider the integral of $g_{1}$. Performing the $r$ integral, we get

$$
\int_{0}^{L} d x^{\prime} \int_{0}^{\infty} d r g_{1}=\sum_{m=-\infty}^{\infty} \int_{0}^{L} d x^{\prime} \frac{x^{\prime}+L m}{24 \pi^{2}\left|x^{\prime}+L m-x\right|^{3}}
$$

where $\|$ denotes the absolute value. Thus one needs to discuss the cases $m \geq 1, m \leq-1$, and $m=0$, respectively. After some calculations, we derive the current for $m \neq 0$ 


$$
j_{2}=-\frac{B\left(L^{2}-4 L x+2 x^{2}\right)}{48 \pi^{2} x(L-x)^{2}}
$$

As for the case $m=0$, the integral (69) is divergent. However, the divergence can be canceled by the integral of $g_{2}$ with $m_{1}=m_{2}=0$. Combining together the integrals of $g_{1}$ with $m=0$ and $g_{2}$ with $m_{1}=m_{2}=0$, we get a finite current

$$
j_{3}=-\frac{B\left(L^{3}(6 \chi+1)+8 L^{2} \chi x-2 L \chi x^{2}-4 \chi x^{3}\right)}{48 \pi^{2} L x(L+x)^{2}} .
$$

Now let us turn to the most complicated parts, the contributions from $g_{2}$ (67). After the radial integration, we have

$$
\int_{0}^{\infty} d r g_{2}=\frac{-x^{\prime}}{3 \pi^{2}} \sum_{m_{1}, m_{2}=-\infty}^{\infty}\left(\frac{1}{\left(\left|A_{1}\right|+\left|B_{1}\right|\right)^{3}}+\frac{1}{\left(\left|A_{2}\right|+\left|B_{2}\right|\right)^{3}}+\frac{\chi}{\left(\left|A_{1}\right|+\left|B_{2}\right|\right)^{3}}+\frac{\chi}{\left(\left|A_{2}\right|+\left|B_{1}\right|\right)^{3}}\right) .
$$

One should discuss cases $m_{1} \geq 1, m_{1} \leq-1, m_{1}=0$ and $m_{2} \geq 1, m_{2} \leq-1, m_{2}=0$, respectively. We have already considered the case $m_{1}=m_{2}=0$ in (71). The other eight cases contribute a current

$$
\begin{aligned}
j_{4}= & -\frac{B\left(3 \pi L x\left(L^{2}-x^{2}\right)^{2} \cot \left(\frac{\pi x}{L}\right)+\pi^{2} L x^{5}+\pi^{2} x^{6}\right)}{72\left(\pi^{2} L^{2} x(L-x)^{2}(L+x)^{2}\right)} \\
& -\frac{B\left(-3 L^{6}+\left(6+\pi^{2}\right) L^{5} x+\left(6+\pi^{2}\right) L^{4} x^{2}-2 \pi^{2} L^{3} x^{3}-\left(3+2 \pi^{2}\right) L^{2} x^{4}\right)}{72\left(\pi^{2} L^{2} x(L-x)^{2}(L+x)^{2}\right)} \\
& +\frac{B \chi\left(L\left(L(3 L-2 x)-\pi^{2} x(L+x) \csc ^{2}\left(\frac{\pi x}{L}\right)\right)-\pi x \cot \left(\frac{\pi x}{L}\right)\left(L^{2}-\pi^{2} x(L-x) \csc ^{2}\left(\frac{\pi x}{L}\right)\right)\right)}{24 \pi^{2} L^{3} x} .
\end{aligned}
$$

In the above derivations, we first perform the $x^{\prime}$ integral and then the sums for $m_{1}$ and $m_{2}$. Fortunately, the sum takes a special form $\sum_{m_{1}, m_{2}=1}^{\infty} f\left(m_{1}+m_{2}\right)$, which can be transformed into only one sum $\sum_{m=2}^{\infty}(m-1) f(m)$. From (68), (70), (71), (73), we finally obtain the anomalous current for complex scalars in a strip

$$
J^{y_{1}}=B \frac{3 \cot \left(\frac{\pi x}{L}\right)\left(L^{2}(\chi+1)+\pi^{2} \chi x(x-L) \csc ^{2}\left(\frac{\pi x}{L}\right)\right)+\pi L(L-2 x)\left(3 \chi \csc ^{2}\left(\frac{\pi x}{L}\right)+1\right)}{72 \pi L^{3}}+O\left(B^{2}\right) .
$$

Note that we have $J^{y_{1}}=-J_{y_{1}}=-\left(j_{1}+j_{2}+j_{3}+j_{4}\right)$ and the other components of currents vanish. Since the magnetic field is a constant in the strip, the current is expected to be antisymmetric, i.e., $J^{y_{1}}(x)=-J^{y_{1}}(L-x)$. This is indeed the case of (74), which is a strong support of our results. Besides, the above currents have the correct limit (44) near the boundary

$$
J^{y_{1}} \sim \begin{cases}\frac{B}{24 \pi^{2} x}, & \frac{x}{L} \rightarrow 0 \\ \frac{B}{24 \pi^{2}(x-L)}, & \frac{L-x}{L} \rightarrow 0,\end{cases}
$$

for both $\operatorname{DBC} \chi=-1$ and NBC $\chi=1$. This is also a test of our calculations. For the convenience of readers, let us draw a figure for the anomalous current in a strip. Without loss of generality, we set $B=L=1$. As showed in Fig. 1, the current of NBC is larger than the one of DBC.

It is straightforward to generalize the above discussions to higher dimensions. Unfortunately, we do not find a general formula. Instead, we calculate them case by case. Please see below for some examples 


$$
\begin{aligned}
& J_{5 d}^{y_{1}}= \frac{-B}{1024 \pi^{2} L^{4} x^{4}}\left[6 L^{4} \chi(L-x)(L+3 x)-24 L x^{4} \zeta(3)(L-2 x)+2 \pi^{2} x^{4}\left(L x-2 \pi^{2} \chi x^{2}\right) \csc ^{2}\left(\frac{\pi x}{L}\right)\right. \\
&+ 2 \pi^{2} x^{4} \csc ^{2}\left(\frac{\pi x}{L}\right)\left(L^{2}\left(2 \pi^{2} \chi+\chi-2\right)+3 \pi^{2} \chi\left(x^{2}-L^{2}\right) \csc ^{2}\left(\frac{\pi x}{L}\right)+2 \pi L(\chi+1) x \cot \left(\frac{\pi x}{L}\right)\right) \\
&+ x^{4}\left(2 L(L(\chi+4)-x) \psi^{(1)}\left(1-\frac{x}{L}\right)-2 L(3 L \chi+x) \psi^{(1)}\left(\frac{x}{L}\right)+2 L(3 L \chi-5 \chi x+x) \psi^{(2)}\left(\frac{x}{L}\right)\right) \\
&\left.+x^{4}\left(\chi(L-x)^{2} \psi^{(3)}\left(1-\frac{x}{L}\right)+\chi(L+3 x)(L-x) \psi^{(3)}\left(\frac{L+x}{L}\right)+2 L(3 L \chi-7 \chi x-x) \psi^{(2)}\left(1-\frac{x}{L}\right)\right)\right] \\
& J_{6 d}^{y_{1}}= \frac{-B}{28800 \pi^{3} L^{5} x^{4}}\left[360 L^{5} \chi(L-x)-8 \pi^{4} L x^{4}(L-2 x)\right. \\
&+30 \pi^{3} L x^{4} \csc ^{2}\left(\frac{\pi x}{L}\right)\left((3 L-x) \cot \left(\frac{\pi x}{L}\right)-4 \pi \chi(L-2 x)\left(3 \csc ^{2}\left(\frac{\pi x}{L}\right)-2\right)\right) \\
&+15 L x^{4}(-2 L \chi-5 L+x) \psi^{(2)}\left(1-\frac{x}{L}\right)+15 L x^{4}(L(2 \chi+5)-x) \psi^{(2)}\left(\frac{x}{L}\right) \\
&\left.+15 \chi x^{5}(L-x)\left(\psi^{(4)}\left(1-\frac{x}{L}\right)-\psi^{(4)}\left(\frac{L+x}{L}\right)\right)\right]
\end{aligned}
$$

where $\psi$ denotes the polygamma function. It is interesting that $\mathrm{DBC} \chi=-1$ and $\mathrm{NBC} \chi=1$ yield different directions of currents in dimensions higher than four. See Fig. 2 for example.

\section{B. Dirac field}

In this subsection, let us investigate the anomalous current of a Dirac field in a strip. For simplicity, we mainly focus on four dimensions. By applying the key formula (31), we get Green's function for the Dirac field

$$
S\left(x^{\prime}, x^{\prime \prime}\right)=S_{0}\left(x^{\prime}, x^{\prime \prime}\right)-B \int_{0}^{L} d x \int_{-\infty}^{\infty} d t d y^{d-2} S_{0}\left(x^{\prime}, x\right) \gamma^{2} x S_{0}\left(x, x^{\prime \prime}\right)+O\left(B^{2}\right)
$$

where

$$
\begin{aligned}
S_{0}\left(x^{\prime}, x^{\prime \prime}\right)= & \sum_{m=-\infty}^{\infty} \frac{(d-2) \Gamma\left(\frac{d-2}{2}\right)}{4 \pi^{\frac{d}{2}}}\left(\frac{\gamma^{0}\left(t^{\prime}-t^{\prime \prime}\right)-\gamma^{1}\left(2 m L+x^{\prime}-x^{\prime \prime}\right)-\gamma^{a}\left(y_{a}^{\prime}-y_{a}^{\prime \prime}\right)}{\left[\left(2 m L+x^{\prime}-x^{\prime \prime}\right)^{2}+\left(y_{a}^{\prime}-y_{a}^{\prime \prime}\right)^{2}-\left(t^{\prime}-t^{\prime \prime}\right)^{2}\right]^{\frac{d}{2}}}\right. \\
& \left.+\chi \cdot \frac{\gamma^{0}\left(t^{\prime}-t^{\prime \prime}\right)-\gamma^{1}\left(-2 m L-x^{\prime}-x^{\prime \prime}\right)-\gamma^{a}\left(y_{a}^{\prime}-y_{a}^{\prime \prime}\right)}{\left[\left(2 m L+x^{\prime}+x^{\prime \prime}\right)^{2}+\left(y_{a}^{\prime}-y_{a}^{\prime \prime}\right)^{2}-\left(t^{\prime}-t^{\prime \prime}\right)^{2}\right]^{\frac{d}{2}}}\right) .
\end{aligned}
$$

Substituting (78), (79), (56) into (52), we get the renormalized current

$$
J^{y_{1}}=B \int_{0}^{L} d x^{\prime} \int_{0}^{\infty} d r\left(h_{1}+h_{2}\right)+O\left(B^{2}\right)
$$

where

$$
\begin{gathered}
h_{1}=-\sum_{m=-\infty}^{\infty} \frac{4 r^{2}\left(L m+x^{\prime}\right)\left(3\left(L m+x^{\prime}-x\right)^{2}+r^{2}\right)}{3 \pi^{3}\left(\left(L m+x^{\prime}-x\right)^{2}+r^{2}\right)^{4}}, \\
h_{2}=\sum_{m_{1}, m_{2}=-\infty}^{\infty} \frac{4 r^{2} x^{\prime}}{3 \pi^{3}}\left(\frac{3 A_{1} B_{1}+r^{2}}{\left(A_{1}^{2}+r^{2}\right)^{2}\left(B_{1}^{2}+r^{2}\right)^{2}}+\frac{r^{2}-3 A_{2} B_{2}}{\left(A_{2}^{2}+r^{2}\right)^{2}\left(B_{2}^{2}+r^{2}\right)^{2}}\right) .
\end{gathered}
$$




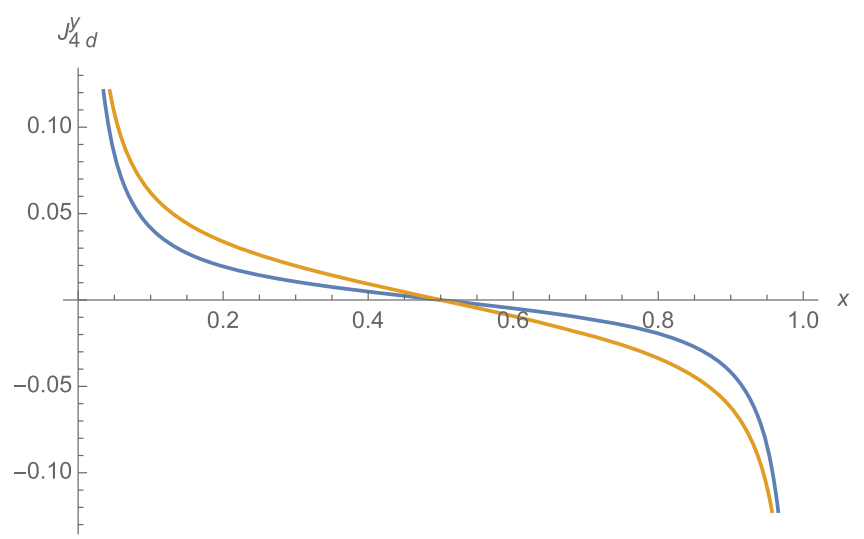

FIG. 1. Anomalous current for scalars in a strip for DBC (blue line) and NBC (yellow line) in four dimensions.

Recall that $A_{1}=2 L m_{1}+x+x^{\prime}, A_{2}=2 L m_{1}+x-x^{\prime}, B_{1}=$ $2 L m_{2}+x+x^{\prime}$, and $B_{2}=2 L m_{2}-x+x^{\prime}$. Following the approaches of Sec. III A, we can derive the current. Since the calculations are similar to those of Sec. III A, we do not repeat them here. We obtain

$$
J^{\mu}=\delta_{y_{1}}^{\mu} \frac{B\left(6 L \cot \left(\frac{\pi x}{L}\right)-\pi L+2 \pi x\right)}{36 \pi L^{2}}+O\left(B^{2}\right)
$$

which obeys the relation $J^{y_{1}}(x)=-J^{y_{1}}(L-x)$ and reduces to the current (59) in a half space in the near-boundary limit

$$
J^{y_{1}} \sim \begin{cases}\frac{B}{6 \pi^{2} x}, & \frac{x}{L} \rightarrow 0 \\ \frac{B}{6 \pi^{2}(x-L)}, & \frac{L-x}{L} \rightarrow 0 .\end{cases}
$$

Similar to the case in a half space, the current (83) in a strip is independent of BCs (49) too. To compare with the currents of scalars, let us draw a figure. From Fig. 3, we notice that the current of the Dirac field is always larger than those of scalars.

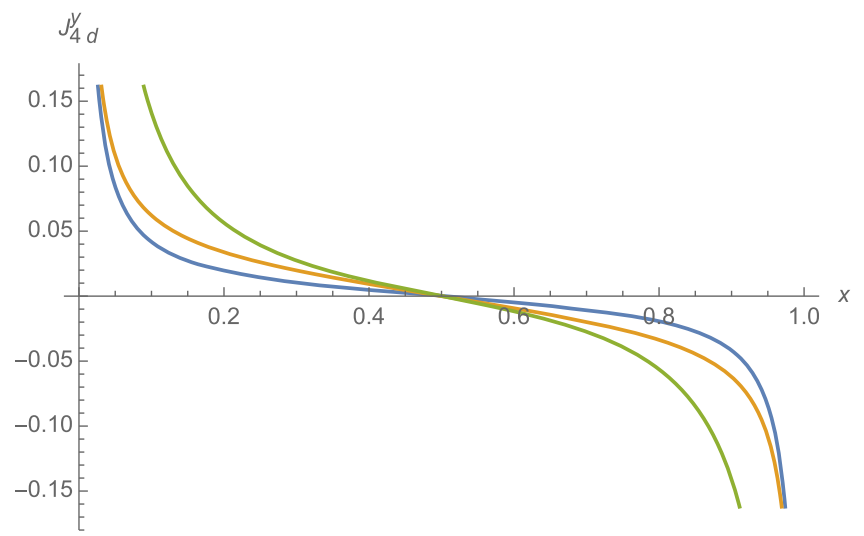

FIG. 3. Current in a strip for Dirac field (green line) and scalars (blue line for DBC and yellow line for NBC) in four dimensions.

The generalizations to higher dimensions are straightforward. Following the above approach, we get anomalous currents for Dirac fields

$$
\begin{aligned}
J_{5 d}^{y_{1}}= & \frac{3}{128 \pi^{2}}\left(\frac{-2 \zeta(3)(L-2 x)-L \psi^{(1)}\left(2-\frac{x}{L}\right)+L \psi^{(1)}\left(\frac{x}{L}\right)}{L^{3}}\right. \\
& \left.-\frac{1}{(L-x)^{2}}\right) B+O\left(B^{2}\right)
\end{aligned}
$$

in five dimensions and

$J_{6 d}^{y_{1}}=\frac{30 L \cot \left(\frac{\pi x}{L}\right) \csc ^{2}\left(\frac{\pi x}{L}\right)-\pi L+2 \pi x}{900 L^{4}} B+O\left(B^{2}\right)$

in six dimensions. Similar to the $4 \mathrm{~d}$ case, currents of Dirac fields are independent of BCs (49) in higher dimensions. Besides, near the boundary, the currents become larger as the dimensions increase. See Fig. 4 for example.

In summary, we have obtained the anomalous currents for complex scalars and Dirac fields in a strip. Our results are exact in the size of strip $L$. In other words, we have got
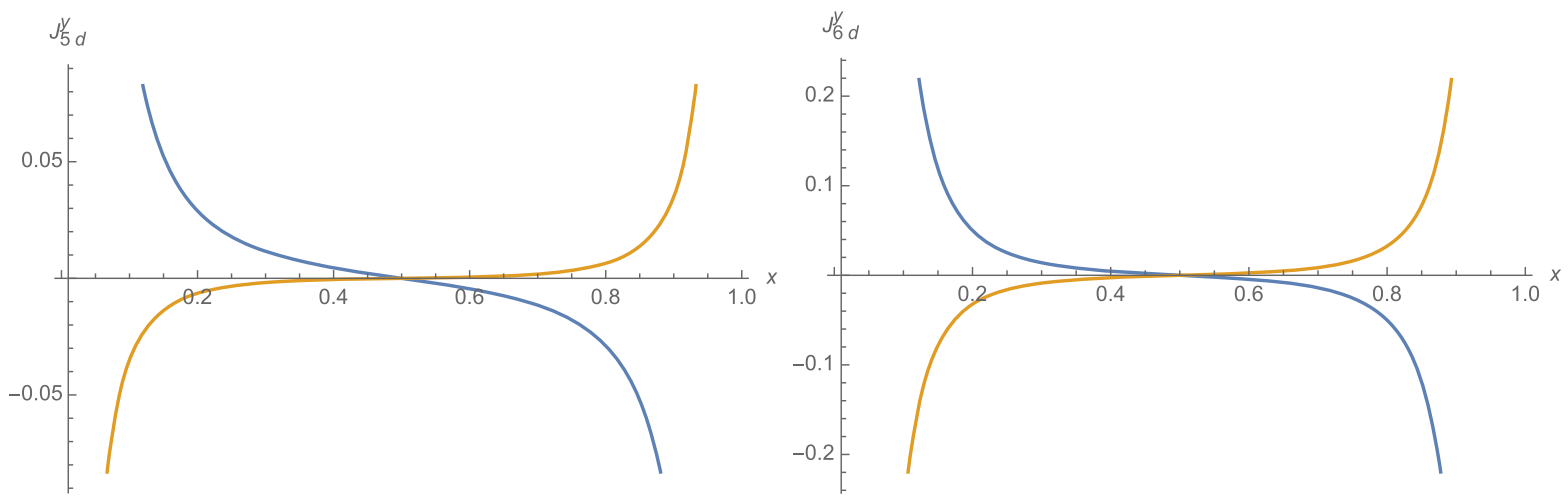

FIG. 2. The left (right) figure denotes 5d (6d) anomalous current for complex scalars in a strip. Blue line is for DBC and yellow line is for NBC. 


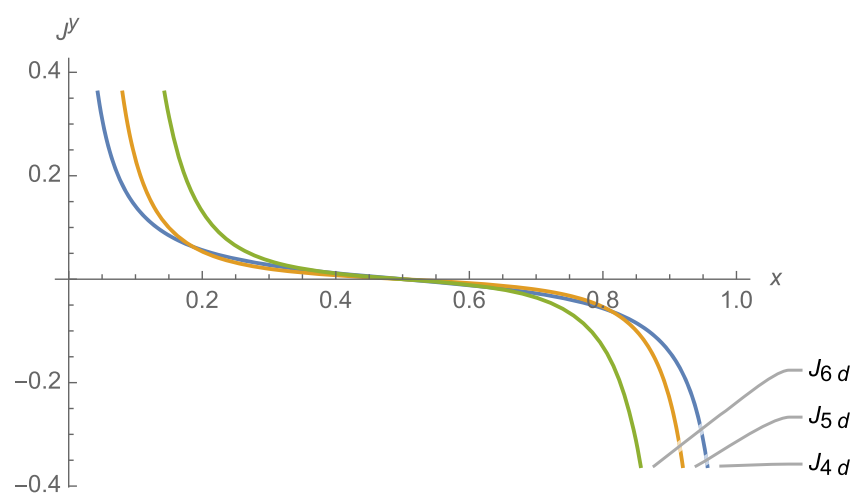

FIG. 4. Anomalous currents of Dirac fields in a strip in 4, 5, 6 dimensions.

the anomalous currents beyond the near-boundary regions of $[17,18]$.

\section{NONPERTURBATIVE RESULTS}

In previous sections, we focus on the anomalous current at the linear order of $O(B)$. In this section, we try to derive the current exactly in the magnetic field $B$. For simplicity, we focus on the complex scalar in a half space with a constant magnetic field $A_{\mu}=(0,0, B x, 0, \ldots, 0)$. We get a formal expression of currents which can be evaluated numerically.

Recall that Green's function obeys EOM

$$
\left[D^{\mu} D_{\mu}+m^{2}\right] G\left(x, x^{\prime}\right)=\delta^{(d)}\left(x, x^{\prime}\right)
$$

where $m$ is the mass. Performing Fourier transform for the tangential coordinates,

$$
G=\int \frac{d k_{\|}^{d-1}}{(2 \pi)^{d-1}} \tilde{G}(k) e^{-i k_{\|} \cdot \Delta x_{\|}}
$$

we can rewrite (87) as

$\left[-\partial_{x}^{2}+\left(m^{2}+k_{\|}^{2}\right)-2 B x k_{1}+B^{2} x^{2}\right] \tilde{G}=\delta\left(x-x^{\prime}\right)$.

Here $k_{1}=k_{y_{1}}, \quad k_{\|} \cdot \Delta x_{\|}=k^{0}\left(t-t^{\prime}\right)-k^{a}\left(y_{a}-y_{a}^{\prime}\right)$, and $k_{\|}^{2}=k_{a}^{2}-k_{0}^{2}$. We split Green's function $\tilde{G}$ into the one in free space and a correction due to the boundary

$$
\tilde{G}=G_{\text {free }}+G_{\text {bdy }},
$$

where $G_{\text {free }}$ is given by $[60,63]$

$$
G_{\text {free }}= \begin{cases}\sqrt{\frac{1}{4 \pi B}} \Gamma\left(\lambda_{k}\right) D_{-\lambda_{k}}\left(\sqrt{2}\left(\bar{x}-\bar{k}_{1}\right)\right) D_{-\lambda_{k}}\left(\sqrt{2}\left(\bar{k}_{1}-\bar{x}^{\prime}\right)\right), & x>x^{\prime}, \\ \sqrt{\frac{1}{4 \pi B}} \Gamma\left(\lambda_{k}\right) D_{-\lambda_{k}}\left(\sqrt{2}\left(\bar{k}_{1}-\bar{x}\right)\right) D_{-\lambda_{k}}\left(\sqrt{2}\left(\bar{x}^{\prime}-\bar{k}_{1}\right)\right), & x<x^{\prime} .\end{cases}
$$

Here $D$ denotes the parabolic cylinder function, $\lambda_{k}=\left(B+m^{2}+k_{\|}^{2}-k_{1}^{2}\right) /(2 B), \bar{k}_{1}=k_{1} / \sqrt{B}$, and $\bar{x}=\sqrt{B} x$. Note that our convention of Fourier transform (88) is different from that of [60]. As a result, $G_{\text {free }}$ (91) differs by a factor $2 \pi$ from the one of [60]. Imposing BCs (8), (9), we solve the corrections to Green's function

$$
G_{\mathrm{bdy}}=\frac{-\Gamma\left(\lambda_{k}\right) D_{-\lambda_{k}}\left(\sqrt{2} \bar{k}_{1}\right)}{2 \pi^{1 / 2} \sqrt{B} D_{-\lambda_{k}}\left(-\sqrt{2} \bar{k}_{1}\right)} D_{-\lambda_{k}}\left(\sqrt{2}\left(\bar{x}-\bar{k}_{1}\right)\right) D_{-\lambda_{k}}\left(\sqrt{2}\left(\bar{x}^{\prime}-\bar{k}_{1}\right)\right)
$$

for DBC and

$$
G_{\mathrm{bdy}}=\frac{\Gamma\left(\lambda_{k}\right)\left(\sqrt{2} D_{1-\lambda_{k}}\left(\sqrt{2} \bar{k}_{1}\right)-\bar{k}_{1} D_{-\lambda_{k}}\left(\sqrt{2} \bar{k}_{1}\right)\right)}{2 \pi^{1 / 2} \sqrt{B}\left(\sqrt{2} D_{1-\lambda_{k}}\left(-\sqrt{2} \bar{k}_{1}\right)+\bar{k}_{1} D_{-\lambda_{k}}\left(-\sqrt{2} \bar{k}_{1}\right)\right)} D_{-\lambda_{k}}\left(\sqrt{2}\left(\bar{x}-\bar{k}_{1}\right)\right) D_{-\lambda_{k}}\left(\sqrt{2}\left(\bar{x}^{\prime}-\bar{k}_{1}\right)\right)
$$

for NBC. Equation (92) for DBC agrees with [60] and (93) for NBC is a new result.

Now we are ready to derive the anomalous current. Substituting (88), (92), (93) into (34) and performing the Wick rotation $k^{0} \rightarrow i k_{E}$ [64], we get the renormalized current

$$
J^{y_{1}}=\int_{-\infty}^{\infty} d p^{d-2} d k_{1} \frac{\left(\bar{x}-\bar{k}_{1}\right) \Gamma\left(\lambda_{p}\right) D_{-\lambda_{p}}\left(\sqrt{2} \bar{k}_{1}\right)}{2^{d-1} \pi^{d-\frac{1}{2}} D_{-\lambda_{p}}\left(-\sqrt{2} \bar{k}_{1}\right)} D_{-\lambda_{p}}\left(\sqrt{2}\left(\bar{x}-\bar{k}_{1}\right)\right)^{2}
$$

for DBC and 

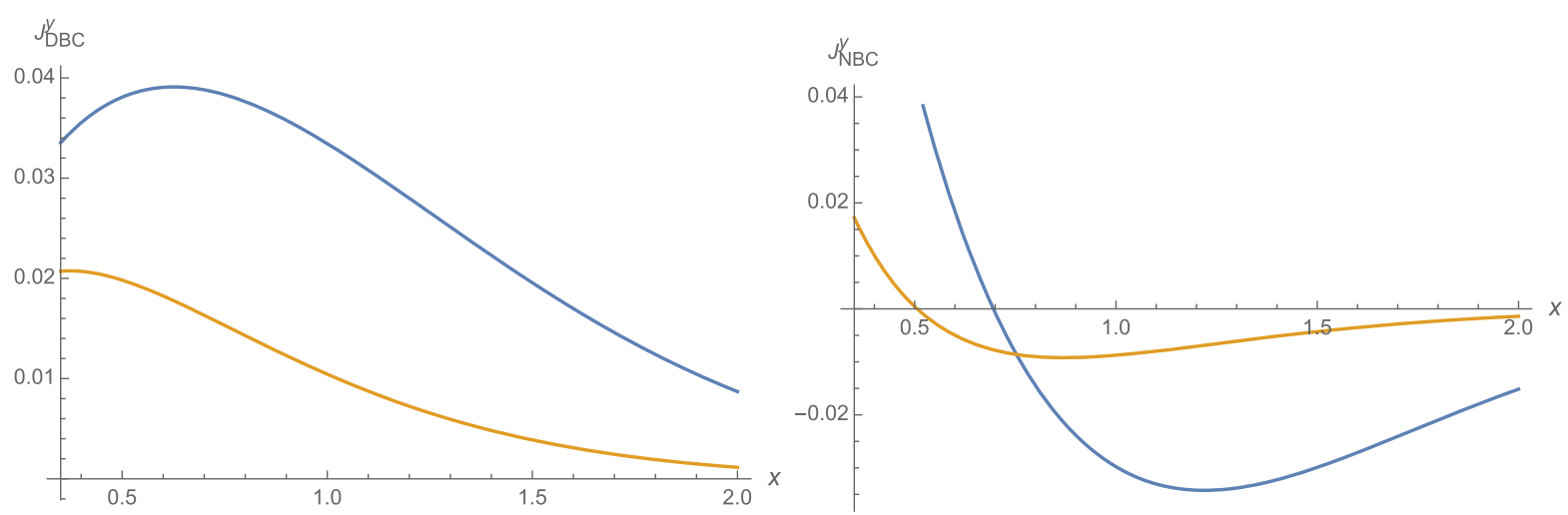

FIG. 5. The left figure is for $2 \mathrm{~d}$ current of DBC and the right figure is for $2 \mathrm{~d}$ current of NBC. Blue line denotes zero mass $m=0$ and yellow line denotes finite mass $m=1$.

$$
J^{y_{1}}=-\int_{-\infty}^{\infty} d p^{d-2} d k_{1} \frac{\left(\bar{x}-\bar{k}_{1}\right) \Gamma\left(\lambda_{p}\right)\left(\sqrt{2} D_{1-\lambda_{p}}\left(\sqrt{2} \bar{k}_{1}\right)-\bar{k}_{1} D_{-\lambda_{p}}\left(\sqrt{2} \bar{k}_{1}\right)\right)}{2^{d-1} \pi^{d-\frac{1}{2}}\left(\sqrt{2} D_{1-\lambda_{p}}\left(-\sqrt{2} \bar{k}_{1}\right)+\bar{k}_{1} D_{-\lambda_{p}}\left(-\sqrt{2} \bar{k}_{1}\right)\right)} \times D_{-\lambda_{p}}\left(\sqrt{2}\left(\bar{x}-\bar{k}_{1}\right)\right)^{2}
$$

for NBC. Recall that $J^{y_{1}}=-J_{y_{1}}, \lambda_{p}=\left(B+m^{2}+p^{2}\right) /$ $(2 B), \bar{k}_{1}=k_{1} / \sqrt{B}$, and $\bar{x}=\sqrt{B} x$. In principle, the formal expressions (94), (95) can be evaluated numerically. Let us take $d=2$ as an example, where the $p$ integral disappears, which can simplify calculations greatly. Note that, for $d=2, B$ of (94), (95) should be understood as the electric field, and $J_{y_{1}}$ should be understood as the charge density. The results are shown in Fig. 5, which implies that the mass suppresses the current and the current approaches zero far away from the boundary. These are the expected behaviors. Note that the numerical integration does not work well near the boundary $x \sim 0$. In the near-boundary region, we can obtain the anomalous current by using methods of Sec. III A. See (45) and (47) for examples.

\section{CONCLUSIONS AND DISCUSSIONS}

In this paper, we have investigated the anomalous current for free theories in the spacetime with boundaries. In a half space, we get the anomalous current at the linear order of magnetic fields in general dimensions. The currents of scalars agree with those in the literature. And the currents for the Dirac field are new. Our results work well in the region close to the boundary, i.e., $B x^{2}<1$. We also obtain the anomalous currents in a strip. The currents are of the linear order of magnetic field $B$ and exact in the size of strip $L$. This means that our results apply to the full region of a strip, as long as the magnetic field is small $B L^{2}<1$. It is remarkable that, unlike the scalar and holographic BCFT, the anomalous currents of free Dirac fields are independent of boundary conditions in general dimensions. It should be mentioned that, although we focus on the constant magnetic field in this paper, our approaches apply to arbitrary electromagnetic fields as well. Finally, we derive a formal expression of anomalous current for complex scalars in a half space. The numerical results imply that the mass suppresses the anomalous currents. In this paper, we mainly focus on dimensions higher than three. It is interesting to study carefully the cases in two and three dimensions. Besides, it is also interesting to study the effect of temperature and the anomalous current with other shapes of boundaries such as cylinders and balls. We hope we could address these problem in the future.

\section{ACKNOWLEDGMENTS}

R.X.M. acknowledges support from NSFC Grant No. 11905297 and Guangdong Basic and Applied Basic Research Foundation (No. 2020A1515010900). 
[1] For a review, see for example, D. E. Kharzeev, Prog. Part. Nucl. Phys. 75, 133 (2014); K. Landsteiner, Acta Phys. Pol. B 47, 2617 (2016).

[2] A. Vilenkin, Astrophys. J. 451, 700 (1995).

[3] A. Vilenkin, Phys. Rev. D 22, 3080 (1980).

[4] M. Giovannini and M. E. Shaposhnikov, Phys. Rev. D 57, 2186 (1998).

[5] A. Y. Alekseev, V. V. Cheianov, and J. Frohlich, Phys. Rev. Lett. 81, 3503 (1998).

[6] K. Fukushima, Lect. Notes Phys. 871, 241 (2013).

[7] D. Kharzeev and A. Zhitnitsky, Nucl. Phys. A797, 67 (2007).

[8] J. Erdmenger, M. Haack, M. Kaminski, and A. Yarom, J. High Energy Phys. 01 (2009) 055.

[9] N. Banerjee, J. Bhattacharya, S. Bhattacharyya, S. Dutta, R. Loganayagam, and P. Surowka, J. High Energy Phys. 01 (2011) 094.

[10] D. T. Son and P. Surowka, Phys. Rev. Lett. 103, 191601 (2009).

[11] K. Landsteiner, E. Megias, and F. Pena-Benitez, Phys. Rev. Lett. 107, 021601 (2011).

[12] S. Golkar and D. T. Son, J. High Energy Phys. 02 (2015) 169.

[13] K. Jensen, R. Loganayagam, and A. Yarom, J. High Energy Phys. 02 (2013) 088.

[14] M. J. Duff, Classical Quantum Gravity 11, 1387 (1994).

[15] M. N. Chernodub, Phys. Rev. Lett. 117, 141601 (2016).

[16] M. N. Chernodub, A. Cortijo, and M. A. H. Vozmediano, Phys. Rev. Lett. 120, 206601 (2018).

[17] C. S. Chu and R. X. Miao, Phys. Rev. Lett. 121, 251602 (2018).

[18] C. S. Chu and R. X. Miao, J. High Energy Phys. 07 (2018) 005.

[19] C. Chu and R. Miao, J. High Energy Phys. 07 (2019) 151.

[20] C. Chu, Fortschr. Phys. 67, 1910005 (2019).

[21] R. Miao and C. Chu, J. High Energy Phys. 03 (2018) 046.

[22] R. Miao, J. High Energy Phys. 07 (2019) 098.

[23] M. N. Chernodub, V. A. Goy, and A. V. Molochkov, Phys. Lett. B 789, 556 (2019).

[24] M. N. Chernodub and M. A. H. Vozmediano, Phys. Rev. Research 1, 032002 (2019).

[25] V. E. Ambrus and M. Chernodub, arXiv:1912.11034.

[26] J. Zheng, D. Li, Y. Zeng, and R. Miao, Phys. Lett. B 797, 134844 (2019).

[27] C. Chu and R. Miao, arXiv:2004.05780.

[28] H. B. G. Casimir, Kon. Ned. Akad. Wetensch. Proc. 51, 793 (1948) [Indagat. Math 10, 261 (1948)]; Kon. Ned. Akad. Wetensch. Proc. 100N3-4, 61 (1997) [Front. Phys. 65, 342 (1987)], http://inspirehep.net/record/24990/export/hlxu.

[29] G. Plunien, B. Muller, and W. Greiner, Phys. Rep. 134, 87 (1986).

[30] M. Bordag, U. Mohideen, and V. M. Mostepanenko, Phys. Rep. 353, 1 (2001).

[31] M. Z. Hasan and C. L. Kane, Rev. Mod. Phys. 82, 3045 (2010).

[32] J. L. Cardy, arXiv:hep-th/0411189.

[33] D. M. McAvity and H. Osborn, Nucl. Phys. B406, 655 (1993).
[34] T. Takayanagi, Phys. Rev. Lett. 107, 101602 (2011).

[35] K. Jensen and A. O'Bannon, Phys. Rev. Lett. 116, 091601 (2016).

[36] D. Fursaev, J. High Energy Phys. 12 (2015) 112.

[37] C. P. Herzog, K. W. Huang, and K. Jensen, J. High Energy Phys. 01 (2016) 162.

[38] C. Herzog, K. W. Huang, and K. Jensen, Phys. Rev. Lett. 120, 021601 (2018).

[39] C. P. Herzog and K. W. Huang, J. High Energy Phys. 10 (2017) 189.

[40] K. Jensen, E. Shaverin, and A. Yarom, J. High Energy Phys. 01 (2018) 085.

[41] M. Kurkov and D. Vassilevich, Phys. Rev. D 96, 025011 (2017).

[42] M. Kurkov and D. Vassilevich, J. High Energy Phys. 03 (2018) 072.

[43] D. Vassilevich, J. High Energy Phys. 07 (2018) 108.

[44] I. Fialkovsky, M. Kurkov, and D. Vassilevich, Phys. Rev. D 100, 045026 (2019).

[45] D. Vassilevich, Mod. Phys. Lett. A 35, 2040017 (2020).

[46] D. Rodriguez-Gomez and J. G. Russo, J. High Energy Phys. 10 (2017) 084.

[47] C. Berthiere and W. Witczak-Krempa, Phys. Rev. B 100, 235112 (2019).

[48] A. Faraji Astaneh, C. Berthiere, D. Fursaev, and S. N. Solodukhin, Phys. Rev. D 95, 106013 (2017).

[49] D. Seminara, J. Sisti, and E. Tonni, J. High Energy Phys. 11 (2017) 076.

[50] J. Erdmenger, M. Flory, C. Hoyos, M. N. Newrzella, and J. M. S. Wu, Fortschr. Phys. 64, 109 (2016).

[51] J. Erdmenger, M. Flory, and M. N. Newrzella, J. High Energy Phys. 01 (2015) 058.

[52] M. Flory, J. High Energy Phys. 06 (2017) 131.

[53] R. X. Miao, C. S. Chu, and W. Z. Guo, Phys. Rev. D 96, 046005 (2017).

[54] C. S. Chu, R. X. Miao, and W. Z. Guo, J. High Energy Phys. 04 (2017) 089.

[55] R. Miao, J. High Energy Phys. 02 (2019) 025.

[56] C. P. Herzog and I. Shamir, J. High Energy Phys. 10 (2019) 088.

[57] C. P. Herzog and I. Shamir, Phys. Rev. Lett. 124, 011601 (2020).

[58] D. M. McAvity and H. Osborn, Classical Quantum Gravity 8, 603 (1991).

[59] D. Deutsch and P. Candelas, Phys. Rev. D 20, 3063 (1979).

[60] P. John and L. G. Suttorp, J. Phys. A 28, 6087 (1995).

[61] L. E. Parker and D. Toms, Quantum Field Theory in Curved Spacetime (Cambridge University Press, Cambridge, England, 2011).

[62] M. Rho, A. S. Goldhaber, and G. E. Brown, Phys. Rev. Lett. 51, 747 (1983).

[63] L. A. Fal'Kovskii, JETP 31, 981 (1970), https://ui.adsabs .harvard.edu/abs/1996tyli.conf...57F/abstract.

[64] M.E. Peskin and D. V. Schroeder, An Introduction to Quantum Field Theory (Addison-Wesley, Reading, USA, 1995). 